David Skålid Amundsen, Camilla Nestande Kirkemo, Andreas Nakkerud, Jørgen Trømborg and Arnt Inge Vistnes. The rainbow as a student project involving numerical calculations. American Journal of Physics 2009, vol 77(9), pp 795-798

This is an author produced version of the article. The original publication is available at American Journal of Physics: http://link.aip.org/link/?ajp/77/795

Access to the published version may require journal subscription.

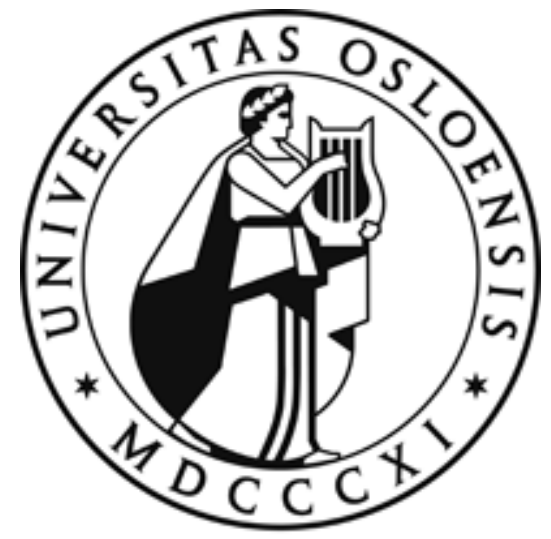




\title{
The rainbow as a student project involving numerical calculations
}

\author{
David Skålid Amundsen, Camilla Nestande Kirkemo, Andreas \\ Nakkerud, Jørgen Trømborg, ${ }^{*}$ and Arnt Inge Vistnes ${ }^{\dagger}$ \\ Department of Physics, University of Oslo, 0316 Oslo, Norway
}

\begin{abstract}
It is a challenge to find interesting and realistic projects where numerical methods can be used to enhance student understanding of physical phenomena. We present such a project in which a group of students used numerical methods to analyze the physics of the rainbow. The project is suitable for students in an undergraduate physics course on the basic principles of geometrical optics. The central part of this paper is written by a group of students, and the introduction and discussion are written by their teacher. In this way both the students' and teacher's perspectives on using numerical methods are presented.
\end{abstract}




\section{INTRODUCTION}

During the past fifty years or so, computers have been used to solve real problems in physics. In the early days of computing, students only used numerical methods at the graduate level. However, with the introduction of personal computers about twenty years ago, the situation has changed.

For about ten years physics teachers developed programs that their students could use to do numerical experiments. These programs can be useful pedagogical tools, but they are usually not aimed at developing students' skills in solving problems numerically.

For the last ten years or so universities have offered separate courses in computational physics in order to teach students how to solve physical problems numerically. These courses are very useful, and there are excellent textbooks available. ${ }^{1}$

Computational physics can be used as a tool for calculations and/or as a tool for learning and gaining an understanding of physics. A combination of these two objectives seems to be most fruitful. Wolfgang Christian and collaborators have developed an open source physics library in Java to ease students' programming, and have written several books that show how to use numerical methods in a wealth of examples in physics. $^{2}$

At the University of Oslo the project "Computers in Science Education" was established some years ago to increase the use of numerical methods in science education. ${ }^{3}$ During the first semester the students learn basic programming in a dedicated course for students in natural science disciplines with significant mathematics components. Many programming tasks in this course are taken from mathematics and physics. In the following the strategy given in Ref. 2 is used not only in a single, dedicated course, but in almost every physics course. An overall better physical understanding is the motivation for our "distributed approach." In addition, we offer dedicated high level courses in computational physics. For various reasons we chose to use Python and Matlab as the programming environments for the basic courses. We introduce $\mathrm{C}++$ (and Perl and Fortran) in the dedicated upperlevel computational physics courses and in the graduate courses.

The use of numerical methods in physics education is beneficial in several respects. Physics students acquire a skill that they will use frequently after they graduate. But even while they are studying, there are numerous positive side effects of using numerical methods in physics courses. We can often easily extend a problem from a standard textbook 
problem to more realistic ones. In this way the problem becomes more interesting and fun for students and teacher. Second, the underlying physics is often more apparent when we solve a problem numerically. The main physical relation is crucial, as well as the initial and boundary conditions. The focus can be on the main principles, instead of on the solution itself.

It is in this context the present work was initiated. The work we will discuss in this article is based on a student project undertaken during the last half of the second year out of a three years undergraduate program. The four students involved worked all day for five days, amounting to approximately 40 hours each, a total of 160 hours. The assignment was short: "Calculate the rainbow using geometrical optics and the dispersion relation of water." It was not stated that numerical methods should be used. The students started by first understanding the physics behind the problem, and which equations they needed to solve and interpret. They developed the necessary computer program along with their investigation of how the system behaved, and wrote an extensive report. In Sec. II the students describe how working with this problem helped them achieve a better understanding of the phenomena.

\section{THE STUDENTS' DESCRIPTION OF THEIR WORK WITH THE PROJECT}

We started out with the law of reflection, Snel's law of refraction, and some geometrical considerations. Fresnel's equations of transmission and reflection coefficients were expected to play an important role in describing the intensity of the light at different angles of deflection.

We approached the problem by sketching the transmission of light rays through a raindrop, using the law of reflection and Snel's law of refraction to find the path of the rays through the drop. To do so we needed to make some assumptions. To apply geometrical optics we assumed that raindrops are spherical. We approximated the incident rays as being parallel, effectively assuming that the Sun is infinitely far away. We needed to consider that light is not really described by rays, but are waves. We assume that the raindrops are sufficiently large compared to the wavelength. This assumption allows us to neglect the curvature of the raindrop at the points of transmission and reflection, which in turn allows the use of laws for reflection off and refraction through flat surfaces. We used Fresnel's 
equations to calculate the intensity of the light transmitted compared to the light reflected at various positions along the drop surface. When we treated the intensity of the light with Fresnel's equations, we assumed that the intensity of the light is not reduced as long as the light propagates in the same medium. Also, we restricted our model to unpolarized incident light.

We started our project by considering the first order rainbow, in which the light undergoes one reflection inside the raindrop. The extension to zeroth and second order rainbows was treated later.

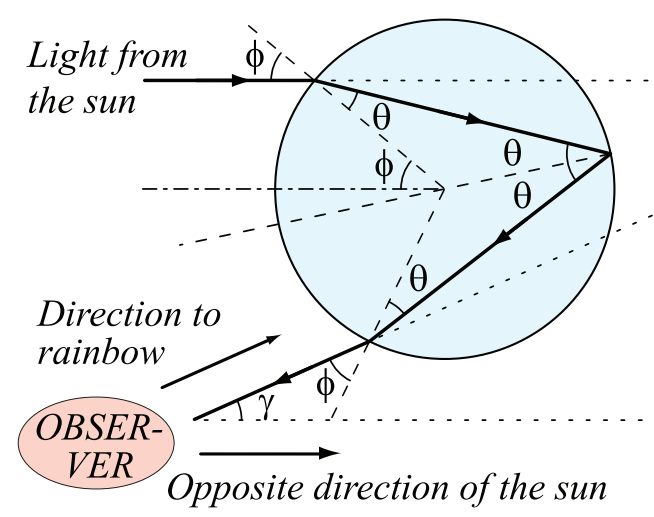

Fig. 1: Illustration of the path of a light ray through a raindrop, defining the angles $\phi, \theta$ and $\gamma$.

Although the incident light rays are parallel, the rays emerging from the raindrop are not. In Fig. 1 one light ray is given for the case that the ray hits the drop at a point where the angle of incidence is $\phi$ (the angle of incidence will depend on where the ray hits the drop, as the incoming rays are parallel). The ray is refracted into the drop, reflected from the back surface, and refracted on its reentry into air. This particular ray leaves the drop at an angle $\gamma$ relative to the direction to the sun, which is parallel to the incoming ray. Rays that hits a raindrop at different points of incidence, and thus with different angles of incidence, will in general be deflected with different values of $\gamma$.

The incident light is a combination of all wavelengths $\lambda$, each with a different index of refraction $n_{\lambda}$; rays enter at every angle $\phi$. The composition of light at each value of $\gamma$ is given by an integral over all $\phi$ and all $\lambda$. A derivation of $\gamma\left(\phi, n_{\lambda}\right)$ for a single ray follows.

A ray that enters the raindrop at a point corresponding to the angle of incidence $\phi$ is refracted into the angle $\theta$ (defined in Fig. 1).

$$
\theta=\arcsin \left(\frac{n_{\text {air }} \sin \phi}{n_{\lambda}}\right) .
$$


The refracted light travels through the raindrop in a straight line and is reflected at the back side. The angle of incidence of light at the back is $\theta$, and so is the angle of reflection at this point. The light then travels through the raindrop in another straight line, is incident at the angle $\theta$, and is refracted into the angle $\phi$. The total deflection of the light, $\gamma$, is (see Fig. 1)

$$
\gamma=4 \theta-2 \phi
$$

If we substitute the value of $\theta$ given by Eq. (1) and set $n_{\text {air }}=1$ for simplicity, we obtain an expression for the deflection of rays that are reflected once:

$$
\gamma\left(\phi, n_{\lambda}\right)=4 \arcsin \left(\frac{\sin \phi}{n_{\lambda}}\right)-2 \phi
$$

Once Eq. (3) was established, elementary numerical methods were invoked to visualize it. Figure 2 shows the deflection $\gamma$ of light of different wavelengths as a function of $\phi$. Figure 2 contains four curves corresponding to different indices of refraction for different wavelengths of the incident light. ${ }^{4}$

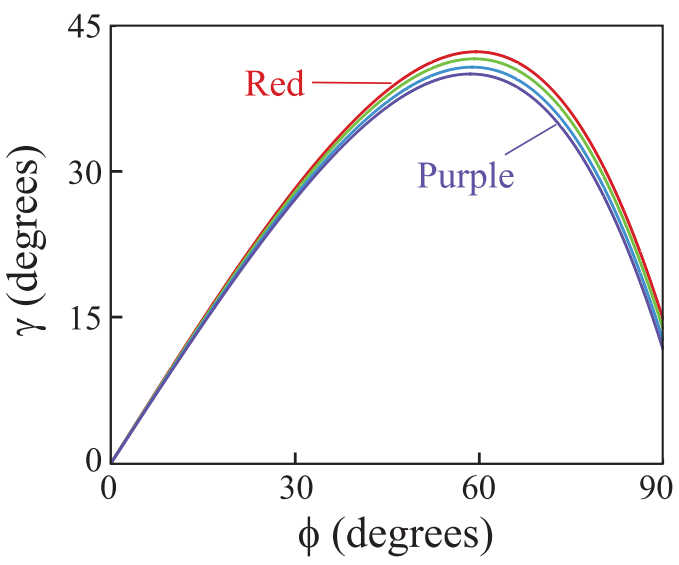

Fig. 2: The angle of deflection $\gamma$ as a function of the angle of incidence $\phi$. The curves correspond to the wavelengths (top to bottom): $650 \mathrm{~nm}, 550 \mathrm{~nm}, 475 \mathrm{~nm}$ and $425 \mathrm{~nm}$. The dots indicate the maximum deflection of each wavelength.

The peaks in Fig. 2 suggests that the position and appearance of rainbows depend on at least two effects. First, there is a maximum angle of deflection, which depends on the wavelength of the incident light. Second, the intensity of the deflected light is higher around the maximum angle of deflection. The first of these observations can be shown using Eq. (3) directly. The second observation is suitable for analytical and numerical analysis. 
Figure 2 reveals that light with different wavelengths may emerge at the same angle $\gamma$. At $\gamma=30^{\circ}$, for instance, we observe light that entered raindrops at $\phi \approx 33^{\circ}$ and $\phi \approx 81^{\circ}$. For this choice of $\gamma$ all wavelengths will contribute roughly equally, thus resulting in white light. To study quantitatively the composition of light at a given $\gamma$, we discretized the $\phi$-axis and calculated $\gamma$ at each discretized value of $\phi$. We made a histogram of all the calculated $\gamma$-values by dividing the $\gamma$-axis into many small intervals $\Delta \gamma$ (as shown in Fig. 3). This histogram represent a numerical integration and is proportional to the intensity of light emerging in the various $\gamma$-directions. The procedure must be repeated for each wavelength. This numerical integration is one of the key challenges of our approach to the problem.

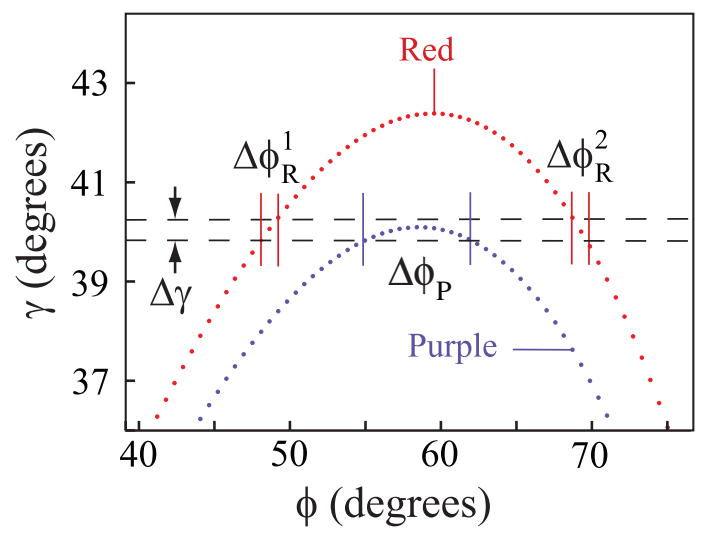

Fig. 3: The idea of our integration method. Light emerging in the interval $\Delta \gamma$ is dominated by purple. The contribution from other wavelengths is much smaller because the purple light will have contributions from an angular interval $\Delta \phi_{P}$ that is much larger than the corresponding intervals for light of other colors (red shown as an example), and therefore $\Delta \phi_{P}$ contains more integration points.

The bin size $\Delta \phi$ must be chosen sufficiently small so that we end up with a sufficiently large number of calculated $\gamma$-values within a chosen interval $\Delta \gamma$. If not, the accuracy of the calculation will be low. We should also carefully consider the placement of the interval in which the maximum value of $\gamma$ occurs. If the maximum is at the middle of a $\gamma$-interval, the upper half of the interval will be empty, causing an error relative to the previous intervals.

The spatial extension (corresponding to the amount of incoming light) of an interval of size $\Delta \phi$ depends on the angle $\phi$ at which it is centered. An interval near $\phi=0$ will intercept a lot of light, while an interval of the same angular extension, but located near $\phi=\pi / 2$, will hardly intercept any light at all. If $h$ is the height of the interval ( $h$ is measured in the 
direction normal to the incoming light), $h$ is given as

$$
h \approx r \cos (\phi) \Delta \phi, \quad(\Delta \phi \ll 1),
$$

where $r$ is the radius of the drop as illustrated in Fig. 4. We will relate the outgoing intensity to the total intensity intercepted by the drop, so that the quantity of interest is $h / r$. At this point, Fresnel's equations can be omitted for simplicity, but only a few lines of code are required to implement them. (As mentioned, Fresnel's equations give a detailed description of the intensity distribution between reflected and refracted light beam at various angles of incidence.) If they are taken into account, the suggested integration scheme yields Fig. 5.

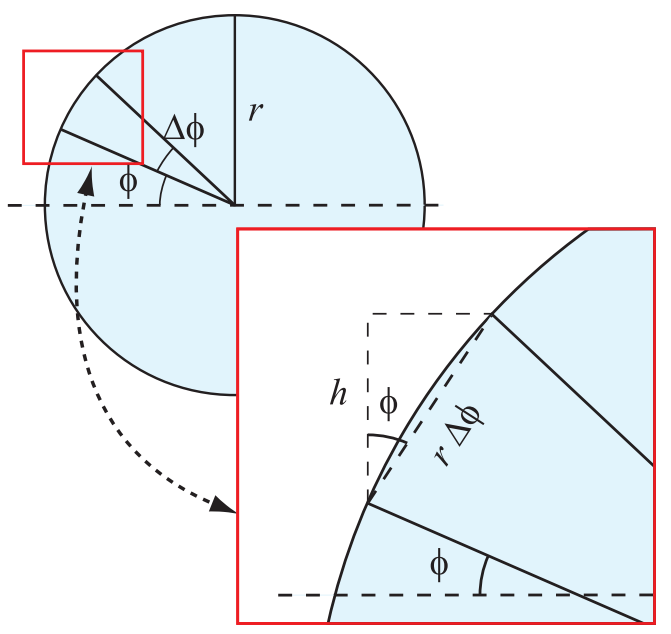

Fig. 4: Illustration of how the spatial extension $h$, and consequently the amount of incoming light in the interval $\Delta \phi$ depends on the angle $\phi$.

In brief, the used integration scheme consists of dividing the $\gamma$-axis into small intervals $\Delta \gamma$, and choosing points in $\phi$ spaced evenly by $\Delta \phi$. For every chosen point in $\phi$, the corresponding value of $\gamma$ is computed. The reflected intensity is weighted by the amount of light entering the drop at the angle $\phi$, given by Eq. (4), and by Fresnel's equations. The total number of contributions to each interval $\Delta \gamma$ is summed. The resulting histogram represents a density distribution of the reflected light. When $\Delta \gamma$ is decreased, the density distribution converge to the sought relation for the reflected intensity at angle $\gamma$.

Both the angles of deflection at which a given color dominates and the total angular extension of the rainbow can be deduced from Fig. 5. Figure 5 also explains the brightness of the area "inside" the rainbow. 


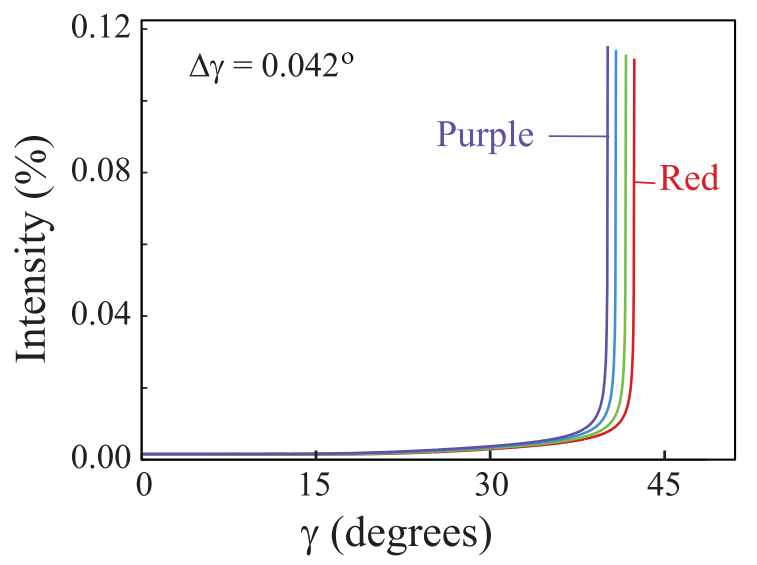

Fig. 5: The intensity of the light emerging at different angles of deflection. An intensity of $100 \%$ corresponds to all light of a given wavelength entering the upper half of the raindrop being reflected into a single outgoing ray. $\Delta \gamma$ is set to $0.042^{\circ}$, one part in thousand of the maximum angular deflection of light entering the raindrop. $\Delta \phi$ is set to $0.00090^{\circ}$, one part in a hundred thousand of a quarter circle.

The intensity of the light that is reflected by one drop is quite low. To see a rainbow it is important to add light from many drops. Fortunately, many drops can contribute. All Sun illuminated drops along a given line of sight from the observer will contribute similarly, irrespective of their distance to the observer.

Our assumptions and the physical principles we applied are inherent to rainbows, and would be part of any treatment of the rainbow. Once Eq. (3) was established, the benefits of a numerical approach became clear. Up to this point in our thinking, we had not considered the use of computers. Looking at Eq. (3) we were at a loss as how to treat this mathematically to obtain an expression that described the rainbow. We could differentiate Eq. (3) and extract the extremum values, but we were not sure what this would tell us. We decided to plot the function to obtain a feel for its behavior.

The plot we obtained is shown in Fig. 2. We first noticed that the maxima are located at different values of $\phi$ and $\gamma$. The significance of this fact was less apparent. From looking at the graph we realized that the flattening at the top of the curves mean that the intensity of light of a given wavelength emerging in an interval $\Delta \gamma$ would be higher near the maximum value of $\gamma$. But we were not sure whether this effect was important enough to explain the separation of the colors. 
Therefore, we wanted to investigate the significance of this "piling up." The integration scheme we described previously gave Fig. 5. Looking back, we can say that it was at this point that we understood the rainbow. Figure 5 shows that the peaks are so dramatically intense and narrow compared to the values for other angles. Thus, the calculations explained why the rainbow is so distinct as we observe in reality.

Because we had chosen wavelengths at each end of the visible spectrum, we also obtained the angular extension of the first order rainbow.

It is straightforward to modify the algorithm to calculate the second order rainbow. Our results explain why the order of the colors is reversed and that the enhanced light intensity on one side is now located on the other side. Thus, it explains the Alexander band. ${ }^{6}$ The relative intensity (compared to the first order bow) is also easily obtained.

When reviewing the outcome of the project, we were inspired and happy to have managed to solve the problem of calculating the rainbow from first principles without any guidelines. The description of the rainbow involves several kinds of problems, which are manageable by second year undergraduate students. We experienced how useful it is to be capable of writing our own small programs. Viewed the other way around, the use of computers helped our understanding of the physics. Particularly enlightening was the creation and evaluation of Fig. 5.

\section{DISCUSSION}

The four students could have followed an analytical analysis similar to the one done by Walker. ${ }^{5}$ In this case the numerical analysis is considerably simpler than the analytical approach. Numerical methods can often be more intuitive for undergraduate students than analytical approaches. It is also easy to discover the significance of different assumptions and relations by simple modifications of numerical algorithms. Furthermore, after one has written a program to solve a simplified version of the problem, it is often straightforward to include a more detailed description of the physics involved. For example, it is simple to implement the use of Fresnel's equations if they were not included initially. It would also be simple to modify the program to handle the polarization of light. It would even be straightforward to implement the changes due to the angular size of the Sun. Such an implementation is far more complicated in an analytic approach. 
Many textbooks give figures like Fig. 3. Such figures are intended to illustrate why the rainbow appears. However, the understanding gained from the generation of Fig. 5 from Fig. 2 is not easily obtained without a hands-on approach.

The programming background of the students doesn't have to be very extensive to solve a problem like the one we have presented here, but the students need to know the basic elements of scientific programming, including the use of variables, arrays, loops, and logical statements. They also need to know how to visualize their data. The program our students wrote to solve this problem was relatively easy to understand. Most students, provided that they have the relevant physics and programming background, should be able to write a program to solve this problem "from scratch."

* Electronic address: jorgen.tromborg@fys.uio.no

$\dagger$ Electronic address: a.i.vistnes@fys.uio.no

1 One excellent example is R. H. Landau, M. J. Pez, and C. C. Bordeianu, A Survey of Computational Physics: Introductory Computational Science (Princeton University Press, Princeton, New Jersey, 2008).

${ }^{2}$ H. Gould, J. Tobochnik, and W. Christian, An Introduction to Computer Simulation Methods (Pearson/Addison-Wesley, San Francisco, CA, 2007), 3rd ed.

3 The project "Computers in Science Education" is described at <www. uhr. no/documents/cse_ nov2008.pdf>.

4 M. R. Querry, D. M. Wieliczka, and D. J. Segelstein, "Water $\left(\mathrm{H}_{2} \mathrm{O}\right)$," in Handbook of Optical Constants of Solids II, edited by E. D. Palik (Academic Press, San Diego, CA, 1998), pp. 10671072.

5 J. D. Walker, "Multiple rainbows from single drops of water and other liquids," Am. J. Phys. 44, 421-433 (1976).

6 The Alexander's band in a rainbow is described at <en.wikipedia.org/wiki/Alexander's_ band>. 